\title{
Operación de Ross en Chile
}

\author{
EDUARDO TURNER G. ${ }^{1}$, RODRIGO MUÑOZ C. ${ }^{1}$, \\ MIGUEL CUMSILLE G. a, SEBASTIÁN ITURRA U. ${ }^{1}$, PABLO STRODTHOFF R. ${ }^{\text {, }}$ \\ NICOLÁS ULZURRÚN T. ${ }^{\mathrm{b}}$, JUAN RODRÍGUEZ A. ${ }^{\mathrm{b}}$
}

\section{Ross operation in Chile}

Background: Donald Ross introduced the pulmonary autograft for aortic valve replacement with reconstruction of the right ventricular outflow tract with a homograft. Despite its advantages over conventional valve prostheses, the Ross Operation is performed in a minority of patients who need an aortic valve replacement throughout the world. Aim: To report the operative and long term results of a series of patients subjected to Ross operation in Chile. Patients and Methods: Between 1996 and 2006, 131 patients aged $35 \pm 11$ years (62\% males) were subjected to an aortic root replacement with a pulmonary autograft and reconstruction of the right ventricular outflow tract with a pulmonary homograft. Seventy percent had congenital valve disease. Associated procedures were done in 39\%. Patients were followed for a mean of $56 \pm 30$ months. Results: Operative mortality was $2.3 \%$. Two patients had the autografts replaced intraoperatively because of tears in the proximal suture line and one within a month of the operation after suffering autograft endocarditis. At last follow up all patients are in functional class 1 or 2. Autograft reoperations were done in two patients who developed dilation with valve regurgitation (both had aortic regurgitation as primary indication for aortic valve replacement). Three patients required reoperation for pulmonary homograft dysfunction. Another three patients had uneventful pregnancies with normal newborns. Actuarial freedom from any reoperation at 10 years is 93\%. Conclusions: The Ross Operation has low operative morbidity and mortality with excellent long term results. Reoperations have been rare within 10 years of follow up both for the autograft or the homograft.

(Rev Med Chile 2010; 138: 413-420).

Key words: Aortic valve; Thoracic surgery; Transplantation, autologous.

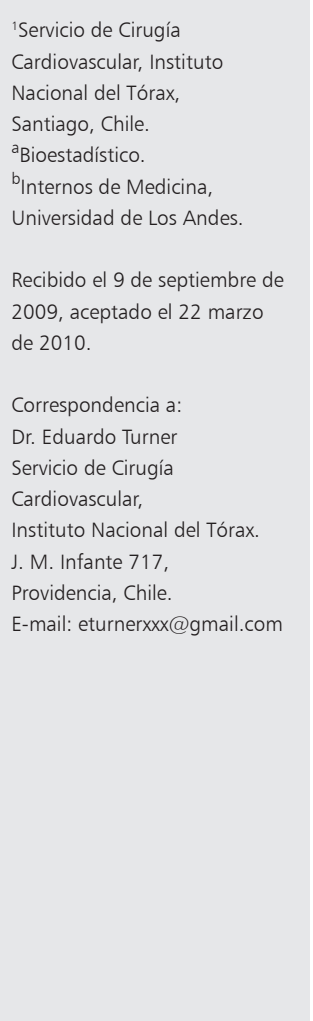

E n el año 1967, el cirujano sudafricano Donald Ross realizó el primer reemplazo valvular aórtico utilizando la válvula pulmonar del paciente (autoinjerto) y reemplazando esta última con un homoinjerto pulmonar ${ }^{1}$ (Figura 1). Previamente, el mismo Ross había iniciado el uso de homoinjertos valvulares aórticos ${ }^{2}$. Sin embargo, en algunos de los pacientes más jóvenes observó un deterioro precoz de las válvulas injertadas que hacía necesario el reemplazo del homoinjerto ${ }^{3,4}$. Ross consideró que la vitalidad $\mathrm{y}$ ausencia de inmunogenicidad del autoinjerto pulmonar aumentarían significativamente la durabilidad de la válvula. Además, esperaba que el homoinjerto pulmonar utilizado para reconstruir la continuidad entre el tracto de salida del ventrículo derecho y la arteria pulmonar, duraría más que en el lado izquierdo por estar sometido a un sistema de presiones más bajas ${ }^{5}$.

La "operación de Ross" o autoinjerto pulmonar posee una serie de ventajas: durabilidad, hemodinamia normal, ausencia de complicaciones tromboembólicas sin necesitar tratamiento anticoagulante y resistencia a las infecciones. Estos beneficios comparados con la necesidad de utilizar tratamiento anticoagulante, riesgo de tromboem- 


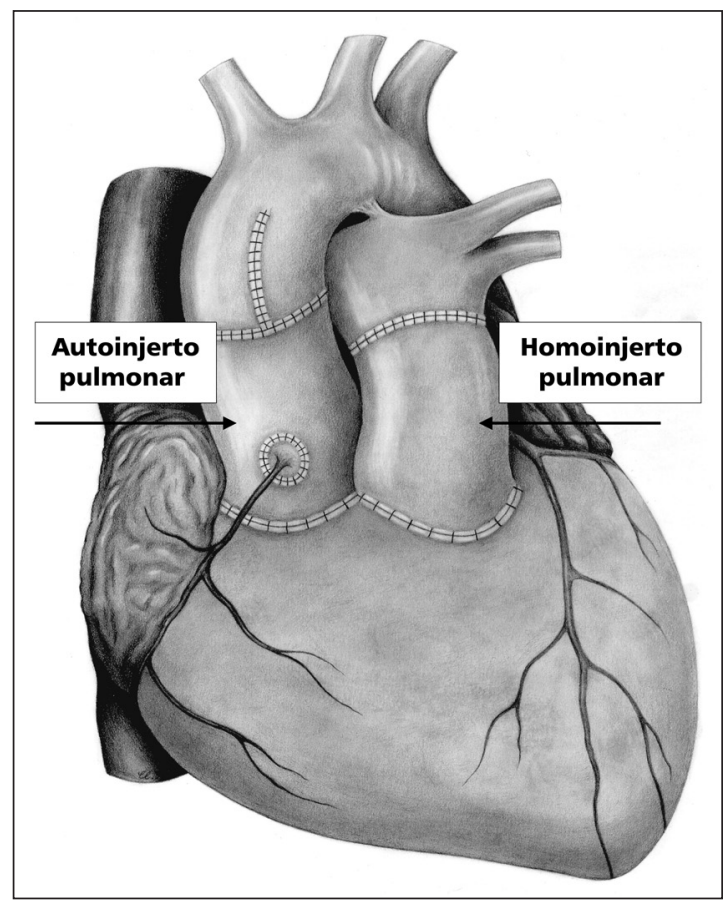

Figura 1. Esquema de operación de Ross.

bolismo en el caso de las prótesis mecánicas, y escasa durabilidad de las válvulas biológicas en pacientes jóvenes ${ }^{6}$ hacen que la operación de Ross sea el procedimiento de elección en este grupo de pacientes ${ }^{7}$. Cuarenta años después, y a pesar de los buenos resultados obtenidos, esta operación es realizada en pocos centros en donde se realiza cirugía cardiovascular. El objetivo de este artículo es reportar los resultados operatorios precoces y el seguimiento a 10 años de un grupo de pacientes con la operación de Ross en Chile.

\section{Pacientes y Métodos}

Durante el período comprendido desde julio de 1996 hasta julio de 2006, a 131 pacientes $(61,6 \%$ hombres; edad promedio $35 \pm 10,7$ años) se les efectuó la operación de Ross. Las características de los pacientes, tipos de lesiones valvulares, capacidad funcional, y tipo de cirugía (electiva o urgencia) están descritas en la Tabla 1. Los pacientes fueron evaluados por el cirujano a cargo, quien propuso la cirugía y discutió su indicación con el paciente o su familiar a cargo en el caso de menores de edad. Todas las cirugías fueron realizadas en 5 hospitales de la Región Metropolitana, $76 \%(\mathrm{n}=99)$ de ellas en el Servicio de Cirugía Cardiovascular del Instituto Nacional del Tórax (INT). La información clínica y peri-operatoria fue obtenida a partir la ficha clínica y de la base de datos de seguimiento prospectivo diseñada para este estudio. El resto de la información fue complementada con datos obtenidos a partir de las fichas clínicas y por medio de contacto directo con los pacientes o sus familiares. Los datos de defunción fueron obtenidos del Registro Civil de Chile.

\section{Técnica operatoria}

Todas las operaciones fueron realizadas con circulación extracorpórea (CEC), hipotermia moderada y administración de aprotinina. La protección miocárdica usada inicialmente fue cardioplegia sanguínea tibia retrógrada y contínua ${ }^{8}$. Luego se cambió progresivamente a cardioplegia sanguínea tibia intermitente anterógrada suplementada por vía retrógrada para optimizar la protección del ventrículo derecho.

En todos los pacientes se hizo un reemplazo de la raíz aórtica (Figura 1): la aorta nativa se secciona en la unión sino-tubular, se reseca el seno de Val-

Tabla 1. Características generales los pacientes operados de Ross $(n=131)$

\begin{tabular}{|lcc|}
\hline & (n) & \% \\
\hline Edad (años) & $35 \pm 11$ & \\
\hline Hombres/Mujeres & $83 / 47$ & \\
Cirugía cardíaca previa & 18 & 14 \\
Etiología: & & \\
Congénita & 92 & 70 \\
Reumática & 22 & 17 \\
Infecciosa & 14 & 11 \\
Lesión & & \\
Estenosis & 58 & 44 \\
Insuficiencia & 35 & 26 \\
Mixta & 38 & 29 \\
Capacidad Funcional & & \\
I & 7 & 5 \\
II & 78 & 60 \\
III & 37 & 28 \\
IV & 9 & 7 \\
Indicación & & \\
Electiva & 123 & 94 \\
Urgencia & 8 & 6 \\
\hline
\end{tabular}


salva posterior y los ostia coronarios son separados y movilizados. Los velos valvulares también se resecan. La raíz es reconstruida con el autoinjerto pulmonar que se sutura directamente al annulus aórtico. Los ostia coronarios son reimplantados a los senos de Valsalva del autoinjerto y el extremo distal de este es anastomosado a la aorta ascendente nativa o un tubo de poliéster en los casos en que se necesita reemplazar la aorta ascendente. La continuidad entre el tracto de salida del ventrículo derecho con la arteria pulmonar es reestablecida con un homoinjerto pulmonar criopreservado o preservado a $4{ }^{\circ} \mathrm{C}$ según disponibilidad.

El tiempo promedio de oclusión aórtica fue 125 $\pm 24,3$ minutos (rango: $90-199$ ) y el de circulación extracorpórea $159 \pm 32$ minutos (rango: 109-324).

En 39\% (52/131) de los pacientes se realizaron procedimientos asociados (Tabla 2 ).

El manejo postoperatorio de los pacientes incluyó el uso de $100 \mathrm{mg}$ de aspirina y beta bloqueadores. Previo al alta se realizó un ecocardiograma de superficie.

\section{Seguimiento}

El control postoperatorio, clínico y ecocardiográfico, fue realizado en el hospital de origen, con el médico tratante o en ambos, al menos una vez al año. Las complicaciones relacionadas con las válvulas implantadas fueron clasificadas de acuerdo a normas internacionales ${ }^{9}$. El seguimiento de los pacientes fue finalizado en julio de 2007.

Estadística: Las variables continuas están expresadas como promedios y desviación estándar. Las variables categóricas se expresan como porcentaje. La sobrevida libre complicaciones valvulares postoperatoria se realizó utilizando el método de

Tabla 2. Procedimientos asociados a la operación de Ross

\begin{tabular}{|lrc|}
\hline & n & \% \\
\hline Reducción de aorta ascendente & 52 & 39,6 \\
\hline Reducción de annulus aórtico (10) & 23 & 17,5 \\
\hline Reemplazo de aorta ascendente & 9 & 6,3 \\
\hline Ensanchamiento de annulus aórtico & 3 & 2,1 \\
\hline Resección de membrana subaórtica & 1 & 0,7 \\
\hline Cierre de comunicación interventricular & 1 & 0,7 \\
\hline Ligadura de ductus & 1 & 0,7 \\
\hline
\end{tabular}

Kaplan-Meier. El análisis estadístico fue realizado utilizando el software SPSS (versión 15.0 para Windows, Inc., Chicago, IL). Los gráficos fueron realizados utilizando el software Graphpad Prism (versión 5.0 para Windows, Graphpad Software, Inc).

\section{Resultados}

\section{Mortalidad y morbilidad perioperatoria}

La mortalidad operatoria fue de 2,3\% (3/131). El primer caso correspondió a un hombre de 29 años que sufrió una falla hepática aguda masiva que se hizo manifiesta a las pocas horas de terminada la operación y cuya causa no pudo ser determinada. La segunda muerte ocurrió en un hombre de 41 años. Su evolución postoperatoria fue sin incidentes. Al momento del alta, el ecocardiograma demostró un derrame pericárdico moderado sin compromiso hemodinámico. Dos semanas después el paciente falleció en un lugar alejado sin que se pudieran obtener datos confiables de lo ocurrido. El tercer caso fue una mujer obesa de 22 años a quien en la infancia se le había hecho una comisurotomía aórtica abierta y más tarde reparación de una coartación aórtica. La operación fue complicada y tuvo un tiempo de oclusión aórtica prolongado (199 minutos). La protección miocárdica fue sólo con cardioplegia retrógrada continua. La paciente falleció debido a falla ventricular derecha irreversible, consecuencia de protección miocárdica insuficiente.

Cinco pacientes $(3,8 \%)$ presentaron complicaciones graves después de descontinuar la CEC. De estos, tres sufrieron isquemia del ventrículo derecho solucionándose con puentes venosos entre la aorta y la coronaria derecha y evolucionando después sin incidentes ni evidencias de infarto perioperatorio. Los otros dos pacientes presentaron sangrado de la sutura proximal del autoinjerto que no se pudo controlar y fue necesario reemplazar el autoinjerto con una prótesis de raíz aórtica porcina "stentless". Uno de estos pacientes requirió de un marcapaso definitivo en el postoperatorio.

Las complicaciones postoperatorias están detalladas en la Tabla 3. Sólo se destaca el caso de un paciente que sufrió una endocarditis del autoinjerto poco antes del mes de la operación y que se complicó con una comunicación interventricular y embolia cerebral. Fue reoperado realizándose un 
Tabla 3. Morbimortalidad perioperatoria de la operación Ross

\begin{tabular}{|lll|}
\hline & n & \% \\
Reoperación por sangrado & 9 & 6,8 \\
\hline Taponamiento cardíaco & 1 & 0,7 \\
Fibrilación auricular & 1 & 0,7 \\
\hline Taquicardia ventricular & 1 & 0,7 \\
Endocarditis & 2 & 1,4 \\
Autoinjerto & 1 & 0,7 \\
Homoinjerto & 1 & 0,7 \\
\hline Déficit neurológico focal & 1 & 0,7 \\
Insuficiencia hepática* & 1 & 0,7 \\
\hline Falla ventricular derecha* & 1 & 0,7 \\
\hline Instalación marcapaso & 1 & 0,7 \\
\hline Desconocida* & 1 & 0,7 \\
\hline
\end{tabular}

*fallecen.

reemplazo del autoinjerto con un homoinjerto aórtico, cierre de la comunicación interventricular y resección de vegetaciones de la válvula tricúspide. Su evolución posterior fue sin incidentes recuperándose ad integrum de su déficit neurológico.

\section{Seguimiento alejado}

El seguimiento de los 128 pacientes restantes fue de $94 \%$ con una media de $56 \pm 30$ meses (rango: 6-125). En este grupo de pacientes no existieron defunciones por lo tanto la sobrevida de esta serie de pacientes a julio de 2007 fue de 98\% (128/131). Todos los pacientes están en ca- pacidad funcional 1 ó 2 . Tres mujeres han tenido embarazos sin complicaciones y partos con recién nacidos normales.

\section{Reoperaciones}

En total, siete pacientes $(5,4 \%)$ debieron ser reoperados por complicaciones relacionadas con el autoinjerto u homoinjerto (Tabla 4). En ambos la indicación quirúrgica original había sido insuficiencia aórtica y no se habían asociado técnicas para reducir el annulus ni la aorta ascendente.

Cuatro $(3,1 \%)$ pacientes fueron reoperados por disfunción del homoinjerto pulmonar. Dos de ellos desarrollaron obstrucción del conducto con velos valvulares indemnes durante el primer año postoperatorio. Ambos casos fueron mujeres. En las dos se amplió el conducto con parches de pericardio bovino reduciendo la obstrucción significativamente. En una de ellas la obstrucción del conducto reapareció y fue necesario reoperarla seis meses más tarde implantándosele una prótesis porcina y reampliando el conducto de forma más extensa. En el tercer caso se detectó insuficiencia progresiva a los pocos meses que se asoció con una dilatación del ventrículo derecho que obligó a reoperarla e implantar otro homoinjerto. El cuarto paciente fue reoperado por endocarditis no controlada con tratamiento antibiótico a los 5 años de la operación. Todos los pacientes evolucionaron sin complicaciones precoces ni tardías.

Al cierre del seguimiento, 96,1\% (123/128) de los pacientes estaban libres de reoperación en el autoinjerto (incluye los dos pacientes a quienes se les reemplazó el autoinjerto en la misma operación) y 96,8\% (124/128) de reoperación del homoinjerto pulmonar (Figura 2).

Tabla 4. Reoperaciones de los pacientes con operación de Ross

\begin{tabular}{|c|c|c|}
\hline Indicación & Tiempo desde cirugía & Tratamiento \\
\hline $\begin{array}{l}\text { Autoinjerto pulmonar } \\
\text { Endocarditis } \\
\text { Dilatación } \\
\text { Dilatación }\end{array}$ & $\begin{array}{l}25 \text { días } \\
65 \text { meses } \\
132 \text { meses }\end{array}$ & $\begin{array}{l}\text { Reemplazo con homoinjerto } \\
\text { Reemplazo con tubo valvulado } \\
\text { Reemplazo con prótesis porcina "stentless" }\end{array}$ \\
\hline $\begin{array}{l}\text { Homoinjerto pulmonar } \\
\text { Insuficiencia } \\
\text { Obstrucción } \\
\text { Obstrucción } \\
\text { Endocarditis }\end{array}$ & $\begin{array}{l}18 \text { meses } \\
18 \text { meses } \\
24 \text { meses } \\
60 \text { meses }\end{array}$ & $\begin{array}{l}\text { Reemplazo con homoinjerto } \\
\text { Ensanchamiento del homoinjerto* } \\
\text { Ensanchamiento del homoinjerto } \\
\text { Reemplazo con homoinjerto }\end{array}$ \\
\hline
\end{tabular}

*reoperada seis meses después por recurrencia de la obstrucción. 


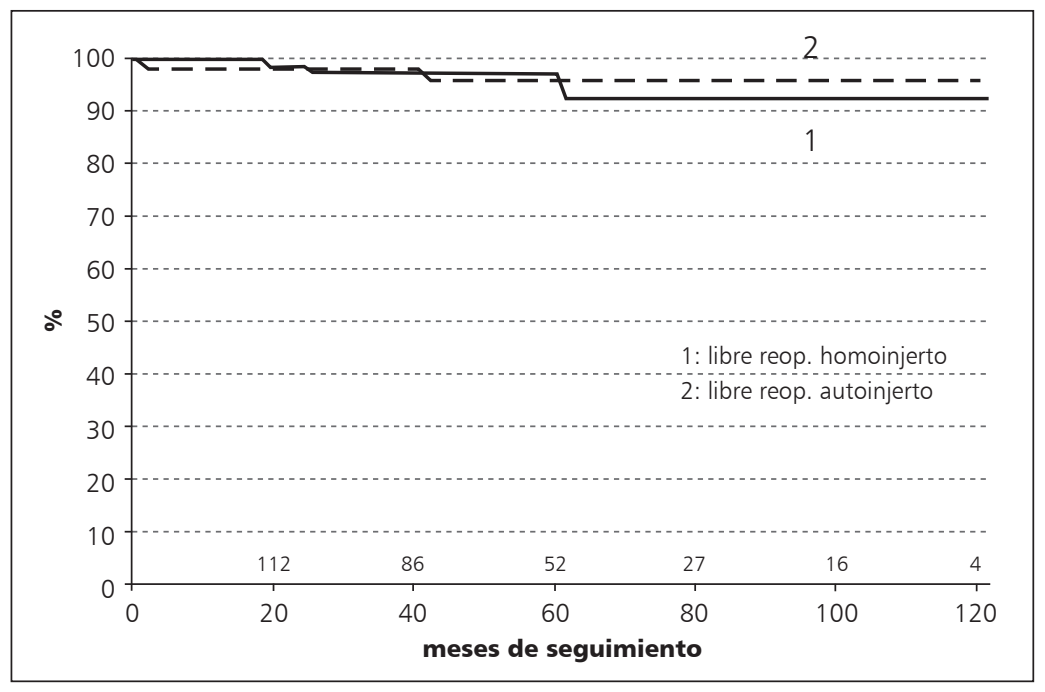

Figura 2. Libertad de reoperación.

\section{Discusión}

La implementación de la Operación de Ross en nuestro medio se puede hacer con resultados operatorios satisfactorios y comparables a los reportados para el reemplazo valvular aórtico con prótesis convencionales ${ }^{11}$. El número de pacientes presentados en esta serie nos permite analizar los problemas de la curva de aprendizaje. A nuestro juicio dos aspectos son fundamentales: 1) experiencia en cirugía de raíz aórtica y 2) protección miocárdica durante el procedimiento.

La experiencia en cirugía de raíz aórtica se consigue con la evolución desde el reemplazo valvular protésico "simple" hasta la ejecución progresiva de procedimientos más complejos en la raíz aórtica.

El segundo, la protección miocárdica, tiene que ver una estrategia quirúrgica adecuada a este tipo de operación. La cardioplegia administrada sólo por vía retrógrada y en forma continua, usada en la fase inicial de la serie, fue parte de una tendencia popularizada a comienzos de la década del $90^{12}$ que probó tener varias desventajas. Las más importantes: protección insuficiente del ventrículo derecho ${ }^{13}$, interferencia con la continuidad de la operación por la presencia de la sangre en el campo operatorio, desplazamiento del catéter de seno coronario haciendo que la solución de cardioplegia no llegue a las coronarias, e hiperkalemia producida por la mayor cantidad de potasio usada. La estrategia actual en base a cardioplegia sanguínea intermitente administrada en forma preferente por vía anterógrada, suplementada por vía retrógrada asegura su distribución uniforme en las coronarias y permite revisar la hemostasia en forma exhaustiva en la raíz aórtica y parte posterior del ventrículo derecho.

Además existen otros factores que explican los buenos resultados operatorios: selección de pacientes predominantemente jóvenes y ausencia de morbilidad asociada.

Para justificar la implementación de una técnica compleja como la operación de Ross, es necesario que los resultados a largo plazo sean mejores que las alternativas actualmente utilizadas en la mayoría de los reemplazos valvulares aórticos en el mundo: las prótesis mecánicas y "biológicas".

Las prótesis mecánicas necesitan tratamiento anticoagulante crónico, lo que implica limitaciones en el estilo y calidad de vida además del control periódico. Esto asociado al riesgo de complicaciones hemorrágicas y tromboembólicas ${ }^{14}$. En países en vías de desarrollo, estas complicaciones son más frecuentes y graves ${ }^{15,16}$.

Las prótesis biológicas en pacientes jóvenes tienen una vida útil limitada, debiéndose reoperar por lo menos $50 \%$ de los pacientes menores de 50 años a los 10 años de la cirugía. Esto fue demostrado convincentemente décadas pasadas ${ }^{17,18}$, de manera que hoy en día se indican principalmente en pacientes mayores de 60 a 65 años en quienes la necesidad de reoperación es probablemente 
menor que la expectativa de vida. Actualmente algunos grupos incentivan el uso de estas prótesis, especialmente las de pericardio bovino fabricada por Edwards basándose en dos ideas: 1) el riesgo de una reoperación es similar al de la primera, y 2) la prótesis en cuestión tiene una durabilidad mayor que las de porcino (producidas por la misma y otras compañías $)^{19,20}$. Sin embargo, en estas publicaciones existen serios conflictos de intereses entre los autores y las compañías que fabrican las prótesis. Este tipo de situaciones ha obligado a las principales revistas científicas a tomar medidas para transparentar esa relación ${ }^{21-23}$. Datos actuales muestran que la durabilidad entre las prótesis biológicas de pericardio de bovino y válvulas aórticas porcinas son comparables ${ }^{24-27}$. Por último, el embarazo, factor importante en pacientes jóvenes, acelera la degeneración de estas prótesis ${ }^{28}$. Con respecto al bajo riesgo de reoperación, sólo es válido para la primera reoperación, aumentando para las siguientes intervenciones ${ }^{29}$.

El reemplazo valvular "convencional" utilizando prótesis biológica o mecánica es un procedimiento relativamente simple que lo convierte en la opción más popular pero puede dejar lesiones residuales importantes: obstrucción significativa en pacientes con anillo aórtico pequeño ${ }^{30} \mathrm{o}$ una raíz aórtica y aorta ascendente dilatada con riesgo de rotura o disección futura ${ }^{31}$. Estos problemas se suelen hacer evidentes en la evolución alejada y requieren de reoperaciones con mayor riesgo ${ }^{32}$.

Los resultados a largo plazo observados en esta serie de pacientes confirman las ventajas de la operación de Ross sobre las prótesis mecánicas y biológicas ${ }^{33}$ : excelente calidad de vida sin necesidad de tratamiento anticoagulante ni limitaciones físicas y riesgo de reoperación muy bajo. Sólo dos de nuestros pacientes han sido reoperados por disfunción del autoinjerto en el seguimiento alejado. Este problema es más frecuente en pacientes que al momento de la cirugía presentan insuficiencia aórtica con annulus y raíz aórtica dilatada ${ }^{34-37}$. Seleccionar pacientes sin esta patología, reducir el annulus, reemplazar la aorta ascendente nativa ${ }^{38}$ $y$ un control estricto de la presión arterial ${ }^{39}$ en el postoperatorio inmediato probablemente minimicen su aparición.

La otra válvula en riesgo en la operación de Ross es el homoinjerto pulmonar usado para reemplazar el autoinjerto. En esta serie 4 pacientes $(3,1 \%)$ debieron ser reoperados por disfunción del homoinjerto. Dos de ellos desarrollaron una estenosis difusa del conducto con velos normales y se presentaron durante los primeros 24 meses. La disfunción precoz es poco frecuente y ocurre generalmente durante los primeros 24 meses $^{40}$. El uso de antiinflamatorios e inmunosupresores ha sido ineficaz en la prevención de esta complicación ${ }^{41}$. En la mayoría de los pacientes el homoinjerto funciona sin problemas por un período prolongado. En adultos la durabilidad del homoinjerto es proporcional a la edad del recipiente. Podría esperarse que pacientes comparativamente mayores no requieran reemplazo del homoinjerto pulmonar en cuyo caso la operación de Ross sería la operación definitiva ${ }^{42}$.

La durabilidad de los homoinjertos pulmonares podría aumentar con el uso de técnicas de ingeniería tisular destinadas a eliminar el componente celular preservando el estroma y repoblando el homoinjerto con células endoteliales del receptor ${ }^{43}$. Las prótesis "stentless" porcinas o las de vena yugular bovina son otras alternativas (estas sólo disponibles en tamaños relativamente pequeños). En ambas los estudios disponibles tienen un número reducido de pacientes y con poco seguimiento, por lo que su rol en la reconstrucción de la continuidad entre el ventrículo derecho y la arteria pulmonar todavía no está definido ${ }^{44,45}$.

Este trabajo tiene limitaciones, 10 años es todavía insuficiente para evaluar largo plazo en cirugía valvular y la ausencia de estudio seriado con imágenes proporcionaría información más precoz y detallada de las válvulas implantadas.

\section{Referencias}

1. Ross DN. Replacement of aortic and mitral valves with a pulmonary autograft. Lancet 1967; 2: 956-8.

2. Ross DN. Homograft replacement of the aortic valve. Lancet 1962; 2: 487.

3. Longmore DB, Lockey E, Ross DN, Pickering BN. The preparation of aortic-valve homografts. Lancet 1966; 2: 463-4.

4. Smith JC. The pathology of human aortic valve homografts. Thorax 1967; 22: 114-38.

5. Ross DN, Sommerville J. Correction of pulmonary atresia with a homograft aortic valve. Lancet 1966; 2: 1446-7.

6. Svensson LG, Blackstone E, Cosgrove DM. Surgical options in young adults with aortic valve disease. Curr Probl Cardiol 2003; 28: 417-80. 
7. Grunkemeier GL, Li HH, Naftel DC, Starr A, Rahimtoola SH. Long-term performance of heart valve prostheses. Curr Probl Cardiol 2000; 25: 73-154.

8. Menasche P, Touchot B, Pradier, F, Bloch G, Piwnica A. Simplified method for delivering normothermic blood cardioplegia. Ann Thorac Surg 1993; 55: 177-8.

9. Edmunds Jr LH, Clark RE, Cohn LH, Grunkemeier GL, Miller DC, Weisel RD. Guidelines for reporting morbidity and mortality after cardiac valvular operations. Ann Thorac Surg 1996; 62: 932-5.

10. Elkins RC, Knott-Craig CJ, Howell CE. Pulmonary autografts in patients with aortic annulus dysplasia. Ann Thorac Surg 1996; 61: 1141-5.

11. Ruel M, Kulik A, Lam B, Fraser DR, Hendry PJ. Longterm outcomes of valve replacement with modern prostheses in young adults. Eur J Cardiothorac Surg 2005; 27 : 425-33.

12. Salerno TA, Houck JP, Barrozo CA, Panos A, Christakis GT, Abel JG, et al. Retrograde continuous warm blood cardioplegia: a new concept in myocardial protection. Ann Thorac Surg 1991; 51: 245-7.

13. Allen B, Winkelmann J, Hanafy H. Retrograde cardioplegia does not perfuse the right ventricle. J Thorac Cardiovasc Surg 1995; 109: 1116-26.

14. Zellner JL, Kratz JM, Crumbley III AJ, Stroud MR, Bradley SM, Sada RM, et al. Long-term experience with the St. Jude Medical valve prosthesis. Ann Thorac Surg 1999; 68: $1210-8$.

15. Villavicencio M, Turner E, Naranjo L. Resultados del Reemplazo Valvular Aórtico con Prótesis Mecánica en el Instituto Nacional del Tórax. Rev Med Chile 2005; 133: 1161-72.

16. Antunes MJ. Should Valve Evaluation be Undertaken in Different Populations from Different Countries?. The Journal of Heart Valve Disease 2004; 13 (Supplement 1): S7-S10.

17. Aupart M, Babuty D, Neville P, Faucher L, Sirinelli A, Marchard M. Influence of age on valve related events with Carpentier-Edwards pericardial bioprosthesis. Eur J Cardiothorac Surg 1997; 11: 929-34.

18. Jamieson WR, Munro AI, Miyagishima RT, Allen P, Burr LH, Tyers GF Carpentier-Edwards Standard Porcine Bioprosthesis: Clinical Performance to Seventeen Years. Ann Thorac Surg 1995; 60: 999-1006.

19. Banbury MK, Cosgrove DM, Thomas JD, Blackstone EH, Rajeswaran, Okies JE, et al. Hemodynamic stability during 17 years of the Carpentier-Edwards aortic pericardial bioprosthesis. Ann Thorac Surg 2002; 73: 1460-5.

20. Smedira NG, Blackstone EH, Roselli EE, Laffey CC, Cosgrove DM. Are allografts the biologic valve of choice for aortic valve replacement in nonelderly patients? Comparison of explantation for structural valve deterioration of allograft and pericardial prostheses. J Thorac Cardiovasc Surg 2006; 131: 558-64.

21. McKneally MF. Beyond disclosure: Managing conflicts of interest to strengthen trust in our profession. J Thorac Cardiovasc Surg 2007; 133: 300-2.

22. Wechsler AS. Conflict of interest: What's a journal to do?. J Thorac Cardiovasc Surg 2006; 131: 771-2.

23. Sade RM. Profits and professionalism. J Thorac Cardiovasc Surg 2002; 123: 403-5.

24. Lund O, Bland M. Risk-corrected impact of mechanical versus bioprosthetic valves on long-term mortality after aortic valve replacement. J Thorac Cardiovasc Surg 2006; 132: 20-6.

25. Yu HY, Ho YL, Chu SH, Chen YS, Wang SS, Lin FY. Long-term evaluation of Carpentier-Edwards porcine bioprosthesis for rheumatic heart disease. J Thorac Cardiovasc Surg 2003; 126: 8-9.

26. Puvimanasinghe JP, Takkenberg JJ, Eijkemans MJ. Comparison of Carpentier-Edwards pericardial and supraannular bioprostheses in aortic valve replacement Eur J Cardiothorac Surg 2006; 29: 374-9.

27. Jamieson WR, Burr LH, Miyagishima RT. CarpentierEdwards supra-annular aortic porcine bioprosthesis: clinical performance over 20 years. J Thorac Cardiovasc Surg 2005; 130: 994-1000.

28. Hung L, Rahimtoola SH. Prosthetic Heart Valves and Pregnancy. Circulation 2003; 107: 1240-6.

29. Davierwala PM, Borger MA, David TE, Rao V, Maganti $\mathrm{M}$, Yau TM. Reoperation is not an independent predictor of mortality during aortic valve surgery. J Thorac Cardiovasc Surg 2006; 131: 329-35.

30. Dumesnil JG, Phibarot P. Prosthesis-patient mismatch and clinical outcomes: the evidence continues to accumulate. J Thorac Cardiovasc Surg 2006; 131: 952-5.

31. Borger MA, Preston M, Ivanov J. Should the ascending aorta be replaced more frequently in patients with bicuspid aortic valve disease?. J Thorac Cardiovasc Surg 2004; 128: 677-83.

32. Szeto WY, Bavaria JE, Bowen FW, Geirsson A, Cornelius $\mathrm{K}$, Hargrove WC, et al. Reoperative aortic root replacement in patients with previous aortic surgery. Ann Thorac Surg 2007; 84: 1592-9.

33. Chambers JC, Somerville J, Stone S, Ross DN. Pulmonary autograft procedure for aortic valve disease: longterm results of the pioneer series. Circulation 1997; 96 (7): 2206-14.

34. Klieverik LM, Takkenberg JJ, Bekkers JA, Roos-Hesselink JW, Witsenburg M, Bogers AJ. The Ross operation: a Trojan horse?. Eur Heart J 2007; 28: 1993-2000. 
35. Luciani GB, Mazzucco A. Aortic root disease after the Ross procedure. Current Opinion in Cardiology 2006; 21: 555-60.

36. Kouchoukos NT, Masetti P, Nickerson NJ, Castner CF, Shannon WD, Dávila-Román VG. The Ross procedure: long-term clinical and echocardiographic follow-up. Ann Thorac Surg 2004; 78 (3): 773-81.

37. Elkins RC, Lane MM, McCue C. Pulmonary autograft reoperation: incidence and management. Ann Thorac Surg 1996; 62: 450-5.

38. Elkins RC, Knott-Craig CJ, Howell CE. Pulmonary autografts in patients with aortic annulus dysplasia. Ann Thorac Surg 1996; 61: 1141-5.

39. Yacoub MH, Klieverik LM, Melina G, Edwards SE, Sarathchandra P, Bogers AJ, et al. An evaluation of the Ross operation in adults. J Heart Valve Dis 2006; 15: 531-9.

40. Carr-White GS, Kilner PJ, Hon JK, Rutledge T, Edwards $\mathrm{S}$, Burman ED, et al. Incidence, location, pathology and significance of pulmonary homograft stenosis after the Ross operation. Circulation 2001; 104: I16-20.
41. Shaddy RE, Hawkins JA. Immunology and failure of valved allografts in children. Ann Thorac Surg 2002; 74: 1271-5.

42. Bohm JO, Botha CA, Hemmer W, Starck C, Blumenstock G, Roser D, et al. Older patients fare better with the Ross operation. Ann Thorac Surg 2003; 75: 796801.

43. Da Costa FD, Dohmen PM, Duarte D, von Glenn C, Lopes SV, Filho HH, et al. Immunological and echocardiographic evaluation of decellularized versus cryopreserved allografts during the Ross operation. Eur J Cardiothorac Surg 2005; 27: 572-8.

44. Kanter KR, Fyfe DA, Mahle WT, Forbess JM, Kirshbom PM. Results with freestyle porcine aortic root for right ventricular outflow tract reconstruction in children. Ann Thorac Surg 2003; 76: 1889-95.

45. Brown JW, Ruzmetov M, Rodefeld, MD, Vijay P, Darragh RK. Valved bovine jugular vein conduits for right ventricular outflow tract reconstruction: an attractive alternative to pulmonary homograft. Ann Thorac Surg 2006; 82: 909-16. 